\title{
SpS1-Preparing for the harvest from large infrared surveys
}

\author{
Deborah L. Padgett ${ }^{1}$ \\ ${ }^{1}$ Spitzer Science Center, California Institute of Technology, M/C 220-6, Pasadena, California \\ 91214, USA \\ email: dlp@ipac.caltech.edu
}

\section{The legacy of IRAS}

During the past decade, there has been a revolution in the availability of multi-wavelength astronomical surveys. From the Sloan Digital Sky Survey (SDSS) to the NRAO VLA Sky Survey (NVSS), astronomical research based on publicly accessible datasets is becoming standard practice in the community. Beginning with the Infrared Astronomical Satellite (IRAS) mission, infrared surveys have played a critical role in stellar astronomy by identifying cool and dusty stars worthy of spectroscopic characterization. IRAS' four photometric bands at $12,25,60$, and $100 \mu \mathrm{m}$ were ideal for detecting dusty circumstellar material. All-sky surveys like IRAS reveal the brightest members of each class of rare objects, optimizing their follow-up strategy. The case of debris disks around main sequence stars demonstrates this utility. IRAS detected dust disks around four nearby stars, Beta Pictoris, Fomalhaut, Epsilon Eridani, and Vega. The "Fabulous Four" remain the best studied debris disks, despite hundreds of additional examples discovered by the SpitzerSpaceTelescope. In the nearly 30 years since IRAS was launched, its highly reliable catalog of just 250000 sources, modest by modern standards, with arcminute scale resolution and 0.3 - 1 Jy sensitivity, has generated over 10,000 references in ADS. This is a success story by any measure.

\section{Ground-based surveys}

Ground-based infrared surveys have led the way in discovering and characterizing brown dwarfs. The Two Micron All Sky Survey (2MASS) mapped the sky in the near-infrared, with sensitivity limits of $J=15.8, H=15.1$, and $K=14.3$. Together with the SDSS and DENIS surveys, 2MASS has determined the space density of ultracool dwarf stars (Reid et al. 2008) and T dwarfs (Metchev et al. 2008). The new UKIRT Infrared Deep Sky Survey (UKIDSS) is approximately five magnitudes deeper than 2MASS, but it only covers a quarter of the sky. Early results include the lowest temperature brown dwarf (550 K; Burningham et al. 2009). The high quality astrometry and photometry in 2MASS makes it an invaluable tool in constructing stellar spectral energy distributions and conducting proper motion studies. The 2MASS catalogs and image server can be found at IRSA (irsa.ipac.caltech.edu), which is also the archive for data from IRAS, Spitzer, and WISE.

\section{Large galactic surveys with Spitzer}

The SpitzerSpaceTelescope is an $85 \mathrm{~cm}$ telescope which operated cryogenically from 2003 to 2005 at wavelengths from 3.6 to $160 \mu \mathrm{m}$. Its warm mission at 3.6 and $4.5 \mu \mathrm{m}$ is ongoing. Although Spitzer is a pointed observatory, it has conducted a number of large "Legacy" surveys of interest to stellar astronomers. Typically, these projects include enhanced data products, such as large mosaics and source catalogs, available via IRSA and the Spitzer Leopard archive tool. These include: 1) imaging surveys of the galactic plane from 3.6 to $70 \mu \mathrm{m}$ : GLIMPSE (PI: Churchwell), MIPSGAL (PI: Carey), GLIMPSE II (PI: Churchwell), MIPSGAL II (PI: Carey), GLIMPSE 3D (PI: Benjamin), SMOG (PI: Carey). Together, these surveys map most of the Milky Way within \pm 5 degrees of the plane. Typical sensitivities for these surveys are tens of microJy at the shortest IRAC bands and 1 mJy at MIPS $24 \mu \mathrm{m}$. 2) imaging surveys of nearby 
star-forming regions from 3.6 to $160 \mu \mathrm{m}$. These include Cores to Disks (c2d; PI: Evans), Taurus 2 (PI: Padgett), Gould's Belt (PI: Allen), and Cygnus-X (PI: Hora). These are imaging maps of individual star-forming clouds with areas of up to 45 square degrees (Taurus). The sensitivity of these maps ( $\sim 10 \mu \mathrm{Jy}$ at the shortest IRAC bands) are higher than the galactic plane surveys due to longer individual exposures. 3) pointed surveys of nearby stars. These include the FEPS survey (PI: Meyer) of several hundred stars from 5 Myr to 5 Gyr and a variety of large guaranteed time and general observer programs which observed many stars within $50 \mathrm{pc}$ for debris disks.

\section{The Wide-field Infrared Survey Explorer (WISE)}

WISE is a $40 \mathrm{~cm}$ telescope which will be cryogenically cooled by solid hydrogen when it is launched into a sun-synchronous orbit in early December 2009. WISE will scan the sky continuously, mapping the entire sky 1.5 times in its ten months of expected cryogenic lifetime. Its large format infrared detectors will produce simultaneous images at $3.3,4.5,12$, and $22 \mu \mathrm{m}$ with resolution of 6 arcseconds ( 12 at $22 \mu \mathrm{m}$ ). The sensitivity is expected to be $0.12 \mathrm{mJy} @ 3.3$ $\mu \mathrm{m}, 0.16 \mathrm{mJy} @ 4.6 \mu \mathrm{m}, 0.85 \mathrm{mJy} @ 12 \mu \mathrm{m}$, and $4 \mathrm{mJy} @ 22 \mu \mathrm{m}$ in the minimum coverage area on the ecliptic. WISE will detect all of the warm debris disks around solar-type stars within $100 \mathrm{pc}$. It will also be the premier tool for finding nearby low temperature brown dwarfs. Predictions indicate that WISE should detect at least a handful of $300 \mathrm{~K} \mathrm{~T}$ dwarfs and quite likely will observe a brown dwarf system closer than Proxima Cen. Information on WISE can be found at www.astro.ucla.edu/ wright/WISE/.

\section{References}

Reid, I. N. et al. 2008, AJ 136, 1290

Metchev, S. A., Kirkpatrick, J. D., Berriman, G. B., \& Looper, D. 2008, Ap.J. 676, 1281

Burningham,B. et al. 2009, MNRAS 395, 1237 